\title{
Autoimmune factors in the ethiopathogenesis of vitiligo
}

\begin{abstract}
Vitiligo is a most common depigmentation disorder, affecting $0,5-1 \%$ world population, caused by loss of functional melanocytes or melanin. Although it is usually asymptomatic, it may cause serious psychosocial problems and impair quality of life. Although many theories about vitiligo etiology exist, its cause still remains unknown. Autoimmune factors seem to have the most important role in the pathogenesis of vitiligo lesions, which is supported by clinical and laboratory data. The main characteristic of vitiligo lesions is the autoimmune destruction of melanocytes and disturbed functional melanocyte-keratinocyte unit, leading to depigmentation. Immune mechanisms involved in vitiligo pathogenesis show prevalence of cellular immune effectors of both innate and adaptive immunity, and their cytokines and chemokines. Better knowledge about their network, and particular role of each protagonist would lead to better therapy, which is often insufficient and by now only symptomatic.
\end{abstract}

Keywords: ethiopathogenesis, vitiligo, depigmentation, immunomodulatory agents, corticosteroids, biological therapy, cd8+ tcells
Volume I Issue 3 - 2015

\section{Vesna Lukinovic-Skudar}

Department of Physiology and Immunology, University of Zagreb, Croatia,

Correspondence: Vesna Lukinovic Skudar, Department of Physiology and Immunology, University of Zagreb, Croatia, Tel +385 I 4590246, Fax +385 I4590207, Email vesnas@mef.hr

Received: October 23, 2015 | Published: October 30, 2015
Abbreviations: APS, autoimmune polyglandular syndrome; APC, antigen-presenting cells; ROS, reactive oxygen species

\section{Introduction}

Vitiligo is an acquired depigmentation disorder, characterized by areas of white macules or patches, related to the loss of functioning melanocytes or melanin in the epidermis or mucosa, or/and hair folliculi. The course of disease is mostly slowly progressive. Its average prevalence is $0.5-1 \%$ worldwide, in all skin types, and first symptoms usually appear before the age of $20 .^{1}$ Although vitiligo is usually asymptomatic, for its prominent lesions on exposed skin areas it can significantly impair patient's quality of life, causing serious psychosocial implications for affected individuals, especially in patients with darker skin types. It can be presented into four main forms: segmental (SV), non-segmental (NSV), mixed and non-classified. ${ }^{2}$ The far most common type is NSV, which is further divided into several clinical subtypes: generalized, acrofacial, mucosal and universal In its most frequent, generalized or common form of NSV, lesions are presented simetricaly bilaterally. SV has unilateral distribution of lesions which follow dermatome shape, it has earlier onset and rapid, but localized progression. ${ }^{2,3}$ Therapy for vitiligo is mainly symptomatic and includes corticosteroids and immunomodulatory agents, vitamin $\mathrm{D}$ analogs and antioxidans, UVA and UVB phototherapy, laser and surgical therapy. ${ }^{4}$ Biological therapy, like anti-TNF $\alpha$ was recently introduced, and it offers promising results in some indications. ${ }^{5}$ Despite many attempts to investigate different factors which could participate in ethiopathology of vitiligo, its cause still remains enigmatic. It is considered as a multifactorial disease, where interplay of genetic, other intrinsic and environmental factors can contribute to the clinical presentation. Genetic factors certainly play an important role in vitiligo, following non-Mendelian polygenic and multifactorial patterns of inheritance. Among more than 33 genes found to be associated with vitiligo, many of them have an immunoregulatory role and are found to be linked to other autoimmune diseases, but only a few of them control melanogenesis. ${ }^{6,7}$ Other theories that try to better explain the etiology of vitiligo include: neural, biochemical, infective, and autoimmune theory. ${ }^{1,8}$ Autoimmune theory is the most supported by consistent laboratory and clinical data, particulary for NSV. Although it seems that SV, has, a different pathogenic mechanism, ${ }^{1,8}$ there is consensus that in all forms of vitiligo there is a certain level of autoimmune components. ${ }^{3}$ Vitiligo was long considered as a primarily skin disorder, but it became clear that it has to be percieved as a systemic disease because of its multiple comorbidities, especially with autoimmune diseases. The highest association exists between vitiligo and thyroid autoimmune disease. Other autoimmune diseases often associated with vitiligo are Addison's disease, psoriasis, type I diabetes mellitus, alopecia areata, pernicious anemia and rheumatoid arthritis. ${ }^{1}$ Vitiligo can be present as a part of autoimmune polyglandular syndromes type 1 (APS1 or APECED) and 2 (APS2) or other autoimmune syndromes. ${ }^{1,8,9}$ This fact suggests that common pathogenic mechanism may underlie all these disorders.

In vitiligo, prominent features are apoptosis of melanocytes and keratinocytes caused by cytotoxic immune reaction, and breakdown of functional melanocyte-keratinocyte crosstalk required for normal pigmentation. There is convincing evidence that the damage and loss of melanocytes in vitiligo is mediated by autoimmune attack of CD8+ T cells. ${ }^{10,11}$ The trigger for this cell-mediated reaction is still unknown. Current view offers an integrative approach, where modification of melanocyte autoantigen by some environmental (UV, skin trauma), or intrinsic factor (metabolic defects, increased oxidation, stress, infection) in genetically-predisposed individuals could induce breaking of self-tolerance to melanocyte antigens and cytotoxic attack. ${ }^{7,12,13}$ Melanocytes here act as a main protagonists for triggering immune reactions, possibly by activation of Ellipsis danger signals". These signals include: active release of heat-shock protein 70 or chaperone-bound melanocyte peptides, that can start both innate and adaptive immune reaction, via stimulation of professional" antigenpresenting cells (APC). ${ }^{14}$ Recently, it was found that IgGs from vitiligo patients showed stronger binding to reactive oxygen species (ROS)modified thyrosinase in comparison with IgGs from healthy controls, and this has positively correlated with duration of disease, severity and 
patients' age. ${ }^{15}$ Thus, melanocyte auto-antigen modification by ROS may increase immune reactivity toward melanocyte, which connects metabolic/oxidation pathogenesis theory with autoimmune theory. Melanocyte-specific CD8+T-cells cause direct damage of melanocytes by granzyme mechanism and apoptosis after their homing into the skin. ${ }^{10}$ Beside CD8+ T cells, specific CD4+ T cells can be activated and can differentiate into several subpopulations, which amplifies immune reaction and potentiates melanocyte destruction. Th1 subpopulations and their cytokines IFN $\gamma$ and TNF $\alpha$, and chemokines CCL5, CXCL9, 10 and 11 seem to have a dominant role in vitiligo ${ }^{16,17}$ as they recruit and activate CD8+ cells, macrophages, and NK cells, promotingc a ellular immune response and that leads cytotoxicity against melanocytes. Chronic inflammation seems to cause impaired melanocyte growth, apoptosis of lesional keratinocytes, and loss of functional melanocyte-keratinocyte cross-talk. A strong Th1 pattern with an active role of CXCL10 in depigmentation was supported in the study on both mouse model and in skin lesions and serum of human patients. ${ }^{18}$ Predominance of Th1 or cytotoxic phenotype in vitiligo may be the result of a disturbed balance between proinflammatory and regulatory $\mathrm{T}$ cells (Treg). Regulatory $\mathrm{T}$ cells, a subset of CD4+ cells with phenotype CD4+ CD25+Foxp3+, exert their function by active suppression of other immune cells, either directly by cell-cell contact, or via secretion of inhibitory cytokines. Alterations in their number or function can lead to autoimmunity. Both Treg number and function have found to be impaired in vitiligo patients. ${ }^{13}$ However, the exact nature of the inverse relationship between Tregs and cytotoxic $\mathrm{T}$ cells in vitiligo is still unexplained, as proper studies are still lacking. Another subset of CD4+ T cells, Th17 cells, were recently recognized as active participants in melanocyte destruction. Th17 cells are responsible for potentiation of the innate inflammatory response, by secreting cytokines (IL-17, IL-21, IL-22) which recruit neutrophils and macrophages to the site of antigen, activation of keratinocytes in local melanocyte unit and increase of production of IL- $1 \beta$ and TNF $\alpha$. Th17-derived cytokines are found to be elevated in serum and lesions in vitiligo patients. ${ }^{19}$ Beside immune cellular effectors and antibodies (which also can participate at a certain level) a special place in the vitiligo pathogenesis belongs to the role of the cytokine and chemokine network, and other signaling molecules, as they are key modulators of any immune reaction. Th1 and Th17-derived inflammatory cytokines and chemokines (IL-17, IL-21, IL-22) as before mentioned above, and many others secreted by other cells have been shown to have disturbed local tissue or serum levels in vitiligo patients, but the exact role of particular cytokine or chemokine, or their receptors, in vitiligo is still far from understood and it's discussion would exceed the scope of this text. They may be a promising target for therapeutic interventions. Some of them are already in use (like anti-TNF $\alpha$ ) or they are under investigation in humans and animal models of vitiligo. ${ }^{5,20}$

\section{Conclusion}

To conclude, this complicated story about autoimmune protagonists in the destruction of melanocytes in vitiligo, both types of immunity, innate and specific, with complex cytokine and chemokine network are involved in vitiligo lesion formation. Additional data and further investigation are neccessary to provide better insight into this complicated area, as extended knowledge about immune factors involved in vitiligo lesions formation could lead to development of new, more efficient therapy, which for now remains only symptomatic.

\section{Acknowledgements}

None.

\section{Conflict of interest}

Author declares that there is no conflict of interest.

\section{References}

1. Alikhan A, Felsten LM, Daly M, et al. Vitiligo: A comprehensive overview part I: Introduction, epidemiology, quality of life, diagnosis, differential diagnosis, associations, histopathology, etiology, and workup. $J$ Am Acad Dermatol. 2011;65(3):473-491.

2. Taïeb A, Picardo M, VETF members. The definition and assesment of vitiligo: a consensus report of the Vitiligo European Task Force. Pigment Cell Res. 2007;20(1):27-35.

3. Ezzedine K, Lim HW, Suzuki T, et al. Revised classification/ nomenclature of vitiligo and related issues: the Vitiligo Global Issues Consensus Conference. Pigment Cell Melanoma Res. 2012;25(3):E1E13.

4. Felsten LM, Alikhan A, Petronic-Rosic V. Vitiligo: A comprehensive overview part II: Treatment options and approach to treatment. $J \mathrm{Am}$ Acad Dermatol. 2011;65(3):493-514.

5. Webb KC, Tung R, Winterfield LS, et al. Tumour necrosis factor-a inhibition can stabilize disease in progressive vitiligo. Br J Dermatol. 2015;173(3):641-650.

6. Spritz RA. Modern vitiligo genetics sheds new light on an ancient disease. J Dermatol. 2013;40(5):310-318.

7. Czajkowski R, Mecinska-Jundzill K. current aspects of vitiligo genetics. Postepii Dermatol Alergol. 2014;31(4):247-255.

8. Boissy RE, Dell'Anna ML, Picardo M. On the pathophysiology of vitiligo: Possible treatment options. Indian J Dermatol Venereol Leprol. 2012;78(1):24-29.

9. Sheth VM, Guo Y, Qureshi AA. Comorbidities associated with vitiligo: a ten-year retrospective study. Dermatology. 2013;227(4):311-315.

10. Van den Boorn JG, Konijnenberg D, Dellemijn TA, et al. Autoimmune destruction of skin melanocytes by perilesional $\mathrm{T}$ cells from vitiligo patients. J Invest Dermatol. 2009;129(9):2220-2232.

11. Wu J, Zhou M, Wan Y, et al. CD8+ T cells from vitiligo perilesional margins induce autologous melanocyte apoptosis. Mol Med Rep. 2013;7(1):237-241.

12. Colucci R, Dragoni F, Moretti S. Oxidative stress and immune system in vitiligo and thyroid diseases. Oxid Med Cell Longev. 2015;2015:631927.

13. Dwivedi M, Kemp EH, Laddha NC, et al. Regulatory T cells in vitiligo: implications for pathogenesis and therapeutics. Autoimmun Rev. 2015;14(1):49-56.

14. Mosenson JA, Zloza A, Klarquist J, et al. HSP70i is a critical component of the immune response leading to vitiligo. Pigment Cell Melanoma Res. 2012;25(1):88-98.

15. Al-Shobaili HA, Rasheed Z. Oxidized thyrosinase: A possible antigenic stimulus for non-segmental vitiligo autoantibodies. J Dermatol Sci. 2015;79(3):203-213.

16. Harris JE, Harris TH, Weninger W, et al. A mouse model of vitiligo with focused epidermal depigmentation requires IFN- $\gamma$ for autoreactive CD8+ T-cell accumulation in the skin. $J$ Invest Dermatol. 2012;132(7):1869-1876.

17. Antonelli A, Ferrari SM, Fallahi P. The role of Th1 chemokine CXCL10 in vitiligo. Ann Transl Med. 2015;3(S1):S16.

18. Rashighi M, Agarwal P, Richmond JM, et al. CXCL10 is critical for the progression and maintenance of depigmentation in a mouse model of vitiligo. Sci Transl Med. 2014;6(223):223ra23. 
19. Elela MA, Hegazy RA, Fawzy MM, et al. Interleukin IL-17, interleukin 22 and FoxP3 expression in tissue and serum of non-segmental vitiligo: A case-controlled study on eighty-four patients. Eur J Dermatol. 2013;23(3):350-355.
20. Lotti T, Hercogova J, Fabrizi G. Advances in the treatment options for vitiligo: activated low-dose cytokines-based therapy. Expert Opin Pharmacother. 2015;15:1-12. 